\title{
Avaliação epidemiológica do traumatismo craniencefálico no interior do Estado de Sergipe
}

\author{
Carlos Umberto Pereira ${ }^{1}$, Gustavo Cabral Duarte², Egmond Alves Silva Santos ${ }^{3}$
}

Serviço de Neurocirurgia do Hospital Governador João Alves Filho. Aracaju, SE, Brasil

\section{RESUMO}

Objetivo: A maior incidência de traumatismo craniencefálico encontra-se nas zonas rurais. Dessa forma, tornam-se imprescindíveis estudos epidemiológicos que definam as características e a etiologia do traumatismo craniencefálico na zona rural, possibilitando estabelecer condutas, estratégias de prevenção, prioridades em pesquisas e planejamento de recursos, além da disponibilidade de profissionais capacitados e serviços de emergência, juntamente com infra-estrutura e aparelhagem adequadas para o tratamento desta patologia. Este estudo visa avaliar, epidemiologicamente, os casos de traumatismo craniencefálico ocorridos na zona rural do Estado de Sergipe analisando-os quanto à idade, sexo, localização, causas do acidente, exame neurológico, exames complementares, conduta, morbidade e mortalidade. Método: Foram estudados, de forma prospectiva e longitudinal, os pacientes admitidos no Serviço de Neurocirurgia do Hospital Governador João Alves Filho, acometidos por TCE e residentes da zona rural do Estado de Sergipe, entre o período de $1^{\circ}$ de setembro de 2003 e 31 de dezembro de 2004. Ao todo, 470 casos foram estudados. Conclusão: Apesar de esta pesquisa demonstrar que $89 \%$ dos pacientes apresentaram TCE-leve e que $78 \%$ dos pacientes foram liberados após atendimento inicial, o TCE na zona rural não deve ser negligenciado, pois representa um grande problema de saúde pública devido aos altos custos hospitalares com atendimentos, exames complementares, tratamento e reabilitação dos pacientes. Nossos resultados fornecem um ponto de partida para a análise das causas e prevenção do TCE na zona rural

\section{PALAVRAS-CHAVE}

Traumatismo craniencefálico. Epidemiologia. População rural.

\section{ABSTRACT}

Epidemiologic findings of the cranial injury in rural areas of the State of Sergipe, Brazil.

Objective: The highest rates of brain injury are located in the rural areas. So, it is necessary an epidemiological study that can define characteristics and the causes of brain injury in rural area, improving knowledge, prevention strategies, priority in research and planning, besides the professionals and emergency services within local and adequate devices to treat this disease. An epidemiological study of brain injury in the rural areas of the State of Sergipe, Brazil, was made analyzing age, sex, location, causes, neurological status, neuroimaging finding, morbidity and mortality. Method: A prospective and longitudinal study of the patients with craniocerebal trauma admitted to the Serviço de Neurocirurgia do Hospital Governador João Alves Filho, coming form the rural zone of the State of Sergipe, Brazil. During the period between September 1, to December 31, 2004, 470 patients were studied. Conclusion: Despite the results of this study demonstrating that $89 \%$ of the patients presented minor trauma, and that $78 \%$ of the patients were set to hospital discharge after initial attendance, craniocerebral trauma in rural zones is not to be neglected because it represents a high cost public health. Our results may supply a starting point to the analysis of the causes and prevention of the craniocerebral trauma in the Brazilian rural zone.

\section{KEY WORDS}

Brain injury. Epidemiology. Rural area.

Este trabalho foi realizado com auxílio de bolsas Iniciação Científica PIBIC/CAPES

1 Prof. Adjunto Doutor do Departamento de Medicina da Universidade Federal de Sergipe. Neurocirurgião do Serviço de Neurocirurgia do Hospital João Alves Filho. Aracaju, SE

2 Médico. Aracaju, SE

3 Médico residente do Serviço de Neurocirurgia do Conjunto Hospitalar do Mandaqui. São Paulo, SP. 


\section{Introdução}

O traumatismo craniencefálico (TCE) é importante causa de morte e deficiência física e mental, superado apenas pelo acidente vascular cerebral como patologia neurológica com maior impacto na qualidade de vida ${ }^{30}$. Segundo dados do Ministério da Saúde do Brasil cerca de dois milhões de pessoas são internadas a cada ano em hospitais da rede pública, vítimas de traumatismos em geral. O TCE constitui-se na principal causa de óbitos e seqüelas nestes pacientes politraumatizados ${ }^{38}$.

O trauma tem sido considerado como a quarta principal causa de morte nos Estados Unidos, principalmente na faixa etária de 1 a 44 anos, onde o TCE está presente em $50 \%$ das mortes de etiologia traumática ${ }^{14}$. Anualmente meio milhão de pessoas requerem hospitalização devido ao TCE nos Estados Unidos, destas 75 a 100 mil morrem no decorrer de poucas horas após a agressão e 70 a 90 mil apresentam lesão irreversível de alguma função neurológica após lesão cerebral ${ }^{38}$. $\mathrm{O}$ TCE, com ou sem politraumatismo associado, é a mais importante causa de morbimortalidade grave acima dos 45 anos de idade 3 .

Na Alemanha, 9.300 pacientes vítimas de acidentes morrem por ano decorrente de trauma cerebral grave ${ }^{3}$. Aqueles que sobrevivem freqüentemente sofrem de seqüelas debilitantes como epilepsia, distúrbios da fala, déficit motor e sensitivo, distúrbios na personalidade e outras lesões neurológicas irreversíveis. Esses dados mostram a importância do TCE, seu impacto na saúde e no gerenciamento público, já que pacientes acometidos dessa lesão apresentam tempo de tratamento e reabilitação mais prolongado e necessitam de maior tempo de internação, o que se agrava na presença de seqüelas posteriores que o impossibilite de executar suas funções, onerando o sistema de previdência social ${ }^{27}$.

Os acidentes automobilísticos são responsáveis por metade dos casos de TCE, sendo que $72 \%$ destes acidentes estão associados ao consumo de bebidas alcoólicas, relacionado quase sempre à imprudência do motorista e ao excesso de velocidade. Outras causas de TCE são quedas acidentais (21\%), particularmente em crianças e idosos, assaltos e agressões (12\%) e causas relacionadas a esportes e recreação $(10 \%)^{27}$.

Os TCE são relatados como incapacitantes, reforçando a sua importância e seu impacto na saúde pública ${ }^{2,7,12,13,16-18,20,22,23,25,36,37}$. Porém, os estudos epidemiológicos em sua maioria abordam populações urbanas, havendo carência de dados sobre TCE na zona rural. Apesar de sua importância, poucos dados epidemiológicos estão disponíveis para auxiliar em estratégias de prevenção, prioridades em pesquisas e planejamento de recursos. Torna-se, então, necessário um estudo epidemiológico que defina e caracterize o
TCE na zona rural para comparação e contraste, visando uma estrutura diferente na prevenção e tratamento dessa lesão em relação à abordagem nas populações urbanas. Esse estudo torna-se ainda mais necessário devido à etiologia e às diferentes características dos TCE na zona rural, como falta de serviço de atendimento pré-hospitalar e serviços com equipamentos e médicos especializados no seu tratamento.

Devido a estes dados, o presente estudo tem como objetivo avaliar epidemiologicamente os casos de TCE ocorridos em residentes da zona rural do Estado de Sergipe, e que foram admitidos no Serviço de Neurocirurgia do Hospital João Alves Filho (Aracaju-SE), analisando dados como: idade, sexo, localização, causa do acidente, exame neurológico, exames complementares, conduta, morbidade e mortalidade.

\section{Revisão da literatura}

O TCE na zona rural é mais freqüente que na zona urbana, mais grave e possui características próprias ${ }^{26,37}$. O conhecimento das causas específicas para cada região é importante para melhor prevenção, definição de estratégias de tratamento e pesquisa e redução do número alarmante de TCE na zona rural. Soma-se a isso a falta de atendimento pré-hospitalar adequado, que é um dos fatores responsáveis pelas complicações decorrentes do trauma na zona rural.

$\mathrm{O}$ atendimento no local do acidente é de importância fundamental para reduzir a morbimortalidade após o TCE. O atendimento inicial objetiva o socorro imediato com conseqüente redução do dano cerebral secundário e do tempo de encaminhamento até o centro de referência ${ }^{38}$. Este atendimento é realizado em boa parte das grandes cidades do país por equipes de resgate devidamente treinadas e equipadas. Porém, na maioria das cidades brasileiras da zona rural, esse serviço não se faz presente ${ }^{38}$, ou quando faz apresenta-se de forma precária. Apesar disso, hoje podemos observar o interesse em instituir um serviço de regate estadual, que em futuro próximo possa melhorar o prognóstico desta injúria.

Alguns artigos demonstraram que há grande variação na incidência do TCE, que varia de 100 a 3.000 por 100.000 habitantes $^{1}$. Gabella e col..$^{15}$ afirmaram maior tendência da população a sofrer TCE quanto maior for a interiorização. Dado esse confirmado por Woodward e col. ${ }^{37}$, quando relataram que as taxas de hospitalização de residentes da zona rural com TCE no sul da Austrália é 33\% maior que a da população urbana. Zhao e col. ${ }^{39}$ estudaram a epidemiologia do TCE durante dois anos, na República da China, e obtiveram como resultados 
uma incidência de $64,1 \%$ de TCE em pacientes provenientes de zonas rurais, sendo que a mortalidade foi de 3,7/100.000 habitantes.

A porcentagem de pacientes com TCE que morreram em hospitais em cidades no sul da Austrália foi maior que os dos hospitais do interior $(1,4 \%$ e $0,4 \%$, respectivamente) $)^{37}$. No entanto, se as mortes de pacientes transferidos do interior para a cidade forem incluídas, o ajuste da taxa de fatalidade em hospitais no interior aumenta para $1,6 \%$, tornando-se então maior que o da zona urbana ${ }^{37}$. Na Austrália, a mortalidade por TCE é $35 \%$ maior no interior que nas cidades ${ }^{37}$. Grande parte dos pacientes internados por TCE em hospitais do interior permanece menos de 48 horas, pois logo após o atendimento de urgência estes pacientes são transferidos para centros mais especializados a fim de receberem o tratamento definitivo ${ }^{37}$. Residentes de áreas rurais apresentaram os maiores índices de TCE e as maiores taxas de mortalidade, quase duas vezes mais alta que a taxa dos residentes urbanos ${ }^{15,37}$. A alta taxa de mortalidade no interior é decorrente do atraso ocasional do atendimento inicial, do tratamento médico inapropriado e da falta de infra-estrutura hospitalar e laboratorial.

Independente da área, o sexo masculino é duas vezes mais propenso a sofrer TCE que o sexo feminino, devido à maior predisposição daquele sexo aos acidentes $^{15,17}$. Zhao e col. ${ }^{39}$ obtiveram a incidência de 2,5 pacientes do sexo masculino para 1 feminino. $\mathrm{O}$ grupo etário de maior risco encontra-se na primeira e segunda décadas de vida, variando conforme o sexo ou residência (zona urbana ou rural) ${ }^{15,17,22}$. Klauber e col..$^{22}$ observaram o impacto do TCE no adulto jovem, já que este é responsável por $20 \%$ a $40 \%$ das mortes no grupo etário abaixo dos 30 anos.

Um estudo realizado no Estado de São Paulo, pelo Ministério da Saúde, observou que a mais alta incidência de TCE foi no grupo etário de 20 a 29 anos, sendo também muito elevada na faixa etária de 0 a 9 anos, representando a principal causa de morte e de seqüelas em crianças e adultos jovens ${ }^{38}$. Aproximadamente um quarto dos pacientes tinha idade inferior a 15 anos. A taxa de mortalidade para a região estudada foi de 37,99 mortes/100.000 habitantes ao ano. Extrapolando-se esta incidência para o montante da população brasileira chega-se a 57.000 mortes anualmente devido a TCE, sendo a maioria por acidentes automobilísticos ${ }^{38}$. Na rede Sarah de hospitais, em 2001, foram atendidos 344 pacientes com TCE. A idade média foi de 28,9 anos e $75,6 \%$ foram do sexo masculino ${ }^{30}$.

Crianças com idade de 5 anos ou mais novas também fazem parte da faixa etária de risco. Segundo o registro nacional de trauma pediátrico dos Estados Unidos, mais de 30.000 crianças tornam-se permanentemente inválidas anualmente, vitimas de
TCE. Perto da metade $(42,6 \%)$ dos TCE ocorre em passeios públicos, $34,3 \%$ no lar e $6,6 \%$ ocorrem em áreas de recreação ${ }^{27}$.

Independente do grau de gravidade do trauma, o nível ocupacional é relacionado aos resultados do $\mathrm{TCE}^{10}$. Taylor e col..$^{35}$ observaram forte associação entre baixa classe socioeconômica e alta mortalidade por acidente automobilístico, tendo as complicações do TCE como a principal causa.

A etiologia do TCE no interior difere da etiologia nos centros urbanos, o que implica em terapêutica orientada, baseada nas evidências. Jagger e col. ${ }^{17}$ relataram os três mecanismos mais comuns de TCE na zona rural: acidentes com veículos motorizados, quedas acidentais e violência. Metade dos TCE envolvem o uso de álcool, seja por parte da vitima ou da pessoa que causou a injúria ${ }^{27}$. Zhao e col. ${ }^{39}$ obtiveram em seu trabalho como causa de TCE na zona rural: quedas acidentais (33,5\%), acidentes com veículos motorizados (33\%), quedas da própria altura $(15,8 \%)$, agressões físicas $(12 \%)$ e por armas de fogo $(1,4 \%)$. Entre as causas de TCE no interior, o suicídio é também um importante agente $^{10}$, sendo que o praticado por arma de fogo tem incidência de $31 \%{ }^{8}$. Muito utilizadas em zonas rurais, as armas de fogo foram responsáveis por $29 \%$ dos TCE nestas regiões, com a mortalidade de $72 \%$ nestes casos. As principais causas de TCE na zona rural por arma de fogo são: suicídio, acidentes de caça, acidentes não-intencionais e seu uso intencional ${ }^{8}$.

Gabella e col. ${ }^{15}$ referem em seu estudo que a epidemiologia do TCE nas áreas rurais e urbanas pode diferir quanto aos caminhos principais para a sua prevenção, devido às suas etiologias distintas. Nesse mesmo trabalho foi utilizada a divisão da população estudada em área de metrópole, áreas de municípios, áreas rurais não remotas e remotas. Para estes mesmos autores, as principais causas de TCE na zona rural são acidentes automobilísticos, suicídio, acidentes com outros tipos de transporte, acidentes com animais, máquinas e o choque não intencional com uma pessoa ou objeto.

Segundo Johnstone e col. ${ }^{19}$ uma das mais significativas barreiras para se obter melhores resultados de pacientes com TCE no interior são as limitações de profissionais capacitados, de instalações hospitalares e de serviços de atendimento pré-hospitalar.

Dois fatores são determinantes na evolução de pacientes com TCE: a extensão e a natureza do dano cerebral irreversível e o subseqüente desenvolvimento de manifestações do dano cerebral secundário. Este, em princípio, pode ser evitado com procedimentos de monitorização e intervenção terapêutica. Além disso, uma melhor evolução do TCE-grave depende da maior eficiência na conduta e tratamento, visando inibir o dano cerebral secundário ${ }^{3}$. 
O estado neurológico final do paciente com TCE é o somatório da lesão irreversível adquirida por ocasião do trauma inicial e da lesão decorrente de insultos secundários. Por ocasião da lesão inicial, parte do cérebro pode apresentar lesão irreversível e parte pode apresentar grau menor de lesão, cuja recuperação pode ocorrer ou não ao longo de um período de semanas ou meses. Lesões secundárias que determinam o agravamento dos déficits neurológicos do paciente incluem distúrbios sistêmicos, como hipóxia, massas cranianas em expansão e aumento persistente da pressão intracraniana. $\mathrm{O}$ imediato reconhecimento e a prevenção das chamadas lesões secundárias é a conduta que oferece a melhor probabilidade de melhora clínica em um paciente com $\mathrm{TCE}^{14}$.

As lesões primárias são aquelas onde o cérebro sofre contusão, laceração e outras lesões, por ocasião do TCE. Estes mecanismos provocam lesão traumática direta do cérebro através de mecanismo de aceleração e desaceleração do encéfalo em relação ao crânio. Essa aceleração poderá acarretar contusão cerebral, ruptura axonal e laceração das veias da ponte ${ }^{14}$.

As contusões ocorrem em regiões nas quais o cérebro em movimento súbito se choca com a base do crânio, ou quando este sofre afundamento que ocasiona lesão no cérebro subjacente. As áreas de contusão são marcadas por hemorragia, edema e necrose do tecido cerebral. A contusão é clinicamente silenciosa quando restrita às porções do cérebro que não apresentam função clinicamente demonstrável. Essas áreas silenciosas podem tornar-se significativas alguns dias depois do TCE, na medida em que o edema aumenta ${ }^{14}$.

A rotação do cérebro dentro da caixa craniana pode provocar ruptura dos axônios na substância branca, acarretando lesão axonal difusa. Essa lesão acompanhase de edema cerebral virtualmente nulo, e praticamente não existe aumento da pressão intracraniana. Uma tomografia computadorizada (TC) de crânio, realizada imediatamente após uma lesão desse tipo, pode revelar hemorragia no corpo caloso e da superfície súperolateral do tronco cerebral, mas o restante do cérebro parece relativamente normal, ainda que o paciente possa manifestar grave dano neurológico ${ }^{14}$.

O déficit neurológico que se segue a uma lesão cerebral penetrante manifesta-se apenas pela perda da função do cérebro diretamente lesado. A piora tardia do estado neurológico do paciente, após uma lesão desse tipo, é secundária ao desenvolvimento de hemorragias ou de infecções que complicam o quadro e que podem ser induzidas por detritos introduzidos no parênquima encefálico por ocasião do traumatismo. As lesões penetrantes devem ser rapidamente limpas, removendo-se fragmentos ósseos, tecido cerebral necrótico e outros detritos. Feridas por projéteis de alta velocidade pro- duzem uma onda de choque que acarreta uma área de lesão mais ampla do que a do trajeto do projétil ${ }^{14}$.

As lesões secundárias mais comuns em pacientes com TCE são as anormalidades metabólicas. Um paciente inconsciente apresenta diminuição dos reflexos protetores normais, o que pode causar obstrução mecânica da orofaringe ou pneumonia por broncoaspiração. A oxigenação pode ainda ser mais comprometida com a presença de contusão pulmonar, tórax instável e embolia gordurosa. A hipercapnia, embora raramente concomitante ao TCE isolado, acarreta vasodilatação e aumento da pressão intracraniana ${ }^{14}$.

Tem-se tornado cada vez mais evidente que o cérebro lesado pode sofrer danos adicionais por processos secundários à lesão inicial. Embora estejam sendo desenvolvidas intervenções experimentais sobre esses processos, elas são do interesse de qualquer médico que clinica em emergência ${ }^{14}$.

Os pacientes com TCE, após a estabilização dos sistemas respiratório e cardiovascular, devem ter a atenção da equipe médica voltada para o sistema nervoso central. Deve-se ter cuidado em manipular a coluna cervical antes de descartar possíveis fraturas, evitando as lesões secundárias nesta região ${ }^{14}$.

No paciente desperto, deve-se proceder a exame neurológico detalhado, com particular atenção a alterações do estado mental, assimetria do diâmetro pupilar, alterações de força ou do tono muscular, assimetria dos reflexos tendinosos profundos e presença de respostas reflexas patológicas. Em um paciente não-cooperativo ou comatoso, o examinador deve confiar na avaliação dos reflexos, visando detectar alterações focais no sistema nervoso. Atenção especial é dedicada aos padrões respiratórios, diâmetro pupilar e resposta à luz, reflexos oculocefálicos, resposta motora aos estímulos dolorosos e reflexos tendinosos profundos ${ }^{14}$.

A avaliação do estado mental é particularmente difícil de registrar, uma vez que se baseia em parâmetros subjetivos e pode variar de examinador para examinador. A Escala de Coma de Glasgow (ECG) é um método padronizado de avaliar a gravidade dos déficits neurológicos do paciente ${ }^{14}$.

A investigação precoce de pacientes com alto risco de complicação intracraniana melhora consideravelmente o prognóstico, e para isso se tornar possível é preciso que os pacientes sejam encaminhados para unidades de neurocirurgia com o intuito de realizar uma $\mathrm{TC}$ e chegar na unidade o mais rápido possível.

Apesar de ser o melhor exame para o diagnóstico de TCE, a TC depende da avaliação e interpretação sem demora do especialista. Em grandes cidades este serviço está presente durante 24 horas, porém na maioria das cidades da zona rural de países do terceiro mundo nem sempre a TC está presente ${ }^{33}$. 
Com a TC as lesões cerebrais podem ser detectadas antes da compressão cerebral séria tornar-se clinicamente óbvia, facilitando uma intervenção cirúrgica precoce, o que reduz consideravelmente a morbimortalidade ${ }^{33}$. Entre os tipos mais comuns de lesão cerebral, decorrentes do TCE, a concussão cerebral foi responsável por $68,4 \%$, a contusão cerebral $26 \%$ e o hematoma intracraniano $5,6 \%{ }^{39}$.

Andersson e col. ${ }^{1}$ relataram que o tratamento do TCE depende do neurocirurgião, associado ao neurologista, neuropsiquiatra, terapeuta ocupacional e outras especializações, com conhecimento e experiência nas desordens neurológicas e suas conseqüências.

Em princípio, o tratamento do TCE apresenta vários componentes, como a organização e qualidade da conduta pré-hospitalar na cena do trauma, o transporte e admissão no hospital adequado e o diagnóstico e procedimentos terapêuticos, incluindo também a reabilitação. Todos esses componentes servem para evitar ou atenuar o desenvolvimento do dano cerebral secundário ${ }^{3}$.

O tratamento do TCE pode ser feito em dois estágios: agudo ou inicial e tardio. $\mathrm{O}$ tratamento inicial focaliza-se em salvar imediatamente a vida do paciente. Pessoal treinado verifica a permeabilidade das vias áreas, assiste a ventilação e a circulação do politraumatizado. A ressuscitação cardiopulmonar pode fazer-se necessária nesse estágio. O tratamento, então, visa à estabilização do paciente. Vários tipos de TCE exigem conduta cirúrgica. Ela pode ser realizada dentro de horas ou dias após o trauma se um hematoma causa aumento da pressão intracraniana. Durante a fase aguda, o inchaço cerebral é monitorado clinicamente, tomograficamente e tratado adequadamente. $\mathrm{O}$ aumento da pressão intracraniana pode comprimir o tecido cerebral dentro da caixa craniana, causando isquemia cerebral. A maior parte dos edemas regride dentro de horas ou dias, mas poucos minutos com a pressão intracraniana elevada podem causar lesões permanentes ${ }^{27}$.

O tratamento tardio consiste na detecção mais precoce das complicações neurológicas e na sua reabilitação. Esse tratamento está baseado na reabilitação e retorno do paciente à sociedade ${ }^{27}$.

Pessoas que residem nas zonas rurais têm uma taxa de mortalidade relacionada ao trauma maior do que os residentes das zonas urbanas ${ }^{1}$. Zhao e col. ${ }^{39}$ obtiveram em seu trabalho a taxa de mortalidade por TCE na zona rural de 9,7/100.000, maior que na zona urbana pesquisada que foi de 6,3/100.000, isso mostra as diferenças entre estas duas zonas quanto à etiologia, características do trauma e serviços de saúde. Muelleman e col. ${ }^{26}$ observaram em sua casuística que a chance de morrer em uma área rural é três a quatro vezes maior que na área urbana.
O prognóstico do TCE depende de vários fatores como: duração do coma, escore da ECG, duração da amnésia pós-traumática, localização e volume do hematoma e de lesões extracranianas associadas ${ }^{27}$. Grande parte dos autores refere dois fatores distintos como determinantes do prognóstico de TCE: a extensão e a gravidade do dano primário ao cérebro no momento do trauma, e as manifestações do dano cerebral secundário, de origem intra e extracraniana ${ }^{3}$.

\section{Pacientes e métodos}

Foram estudados os pacientes admitidos no serviço de neurocirurgia do Hospital Governador João Alves Filho, acometidos por TCE e residentes da zona rural do Estado de Sergipe, entre o período de $1^{\circ}$ de setembro de 2003 e 31 de dezembro de 2004.

O presente estudo foi prospectivo longitudinal.

Foram analisados segundo o protocolo estabelecido em: identificação, constando de dados do paciente como nome, idade, sexo, número de matrícula, data de admissão e origem.

- Causa do TCE: acidente de automóvel, moto ou outro veículo, quedas de bicicleta, atropelamento, queda acidental, espancamento e outros (ferimento por arma de fogo, ferimento por arma branca, acidentes com animais, desconhecidos e não relatados).

- Gravidade do TCE através do escore da ECG, sendo classificado o TCE em leve (13-15), moderado (9-12) e grave (abaixo de 9).

- Exame neurológico no qual foi observado pelo médico plantonista e/ou neurocirurgião as alterações que o paciente apresentava no ato do atendimento, como alteração do nível de consciência, náuseas e vômitos, cefaléia, diplopia, epistaxe, otorragia, exame normal ou sem descrição.

- Exames solicitados e resultados: radiografias e TC de crânio, juntamente com seu laudo.

- Tratamento e evolução: se o paciente foi liberado para casa, internado ou óbito.

Foram utilizadas informações das fichas de admissão hospitalar e dos prontuários dos pacientes após liberação pela direção do Hospital, mantendo o sigilo de todos os casos estudados.

Para a confecção do texto foi utilizado o software Word XP, e para análise, confecção dos gráficos e tabelas foi utilizado o software Excel XP. Para tabulação dos dados e análise foi utilizado o software Epi Info 2002. 


\section{Resultados}

Foi estudada uma amostra de 470 pacientes vítimas de TCE. A idade variou entre 2 meses e 82 anos com a média de 22 anos e moda de 18 anos (Gráfico 1). Houve maior incidência nas faixas etárias entre 10 e 19 anos e 20 e 29 anos, sendo responsável por $45 \%$ dos casos e que estavam associados a acidentes automobilísticos, agressões físicas e quedas acidentais em relação às outras faixas etárias. $\mathrm{O}$ sexo masculino foi o mais acometido, 344 casos (73\%) e 126 casos (27\%) do sexo feminino (Gráfico 2).

Quanto ao local do trauma, os municípios foram assim distribuídos: Nossa Senhora do Socorro (88); São Cristóvão (41); Itabaiana (36); Lagarto (32); Tobias Barreto (18); Laranjeiras (17); Itaporanga d'Ajuda (16); Estância (16); Carira (12); Ribeirópolis (10); Japaratuba (9); Umbaúba, Salgado e Capela (8); Riachão do Dantas, Carmópolis, Itabaianinha, Monte Alegre e Nossa Senhora da Glória (7); Aquidabã, Barra dos Coqueiros, Macambira, Campo do Brito e Canidé do São Francisco (6); Poço Verde e Simão Dias (5); Tomar do Geru, Nossa Senhora das Dores, Poço Redondo, Pedrinhas, Moita Bonita, Gararu, Muribeca, Propriá, Pirambu, São Domingos, Maruim e Porto da Folha (4); Graccho Cardoso e Boquim (3); Santa Rosa de Lima, Pacatuba, Arauá, Telha, Siriri, Indiaroba, Neópolis, Frei Paulo, Rosário do Catete, Areia Branca e Santo Amaro das Brotas (2) (Tabela 1 e Figura 1).
As principais causas de TCE foram: queda acidental 148 casos; acidentes automobilísticos 145: sendo $98 \mathrm{de}$ moto, 40 de carro e 7 outros veículos. Outras causas foram: atropelamento, 24 casos; queda de bicicleta, 33 casos; espancamento, 29 casos; 91 casos por várias causas, como arma de fogo, acidentes com animais e causas indeterminadas (Gráfico 3). Quanto à gravidade do TCE tivemos 422 (89\%) leve, 36 (8\%) moderado e $12(3 \%)$ grave (Gráfico 4).

$\mathrm{O}$ exame neurológico apresentou-se alterado em 211 casos. Os principais sintomas apresentados foram: alteração do nível de consciência 85 casos, náuseas e vômitos 97 , sonolência 51 , cefaléia 40 , tontura 18 , crise convulsiva 11 , otorragia 12 , epistaxe 8 e diplopia 2 (Gráfico 5). O exame foi considerado normal em 182 pacientes e em 77 casos não havia descrição quanto ao exame na ficha de atendimento hospitalar.

Dentre os exames complementares, a TC foi realizada em 242 casos e demonstrou alterações em 75. A radiografia simples de crânio foi realizada em 239 casos, apresentando-se alterada em 4. Em 44 pacientes não foi necessária realização de exames complementares, apenas avaliação clínica (Gráfico 6).

Com relação ao tratamento e evolução do trauma, 368 pacientes foram liberados para o lar após um determinado período de tempo em observação, 99 foram internados e três evoluíram para óbito (Gráfico 7).

Gráfico 1 - Incidência por idade (em anos)
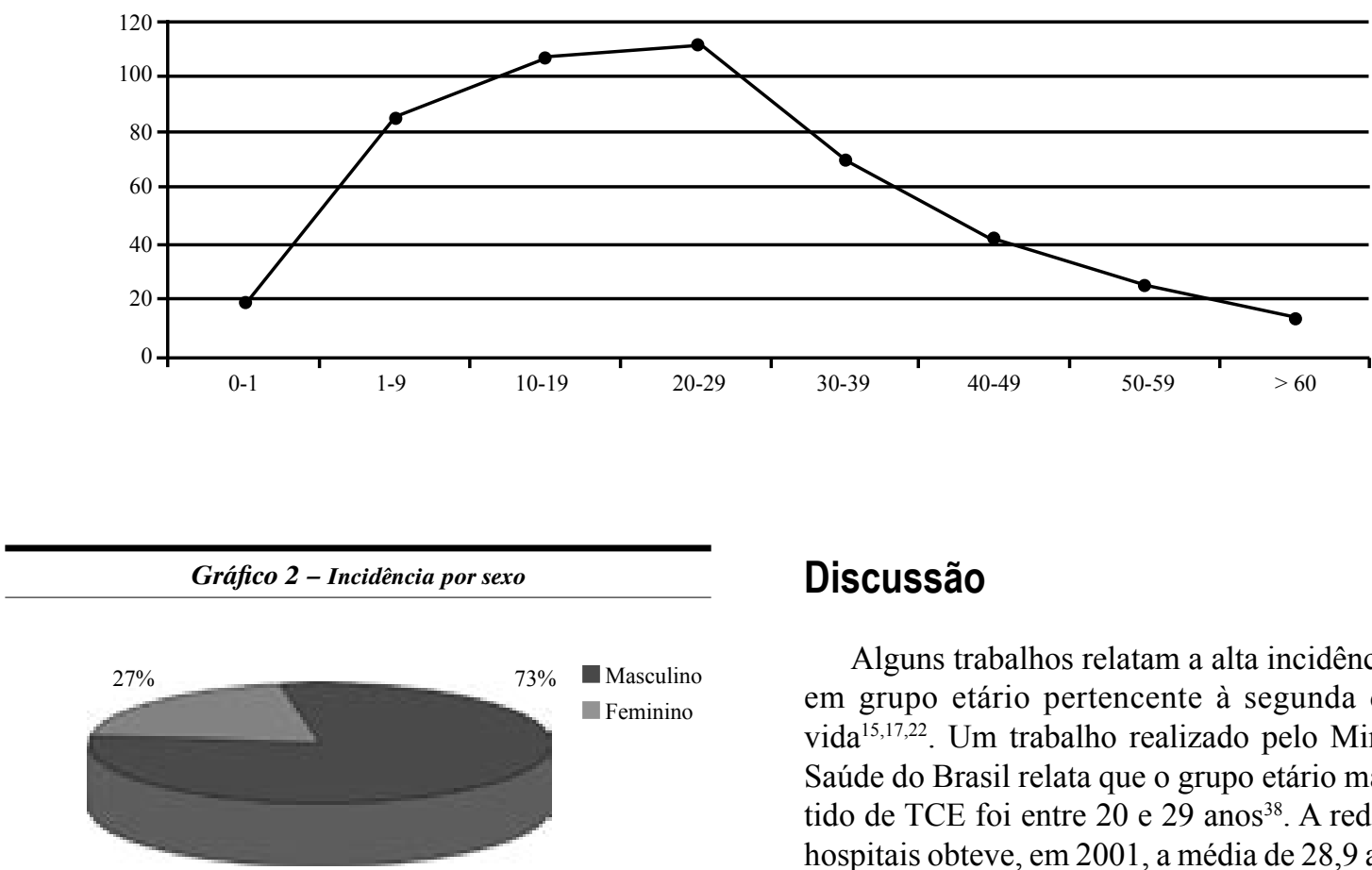

\section{Discussão}

Alguns trabalhos relatam a alta incidência de TCE em grupo etário pertencente à segunda década de vida $^{15,17,22}$. Um trabalho realizado pelo Ministério da Saúde do Brasil relata que o grupo etário mais acometido de TCE foi entre 20 e $29 \operatorname{anos}^{38}$. A rede Sarah de hospitais obteve, em 2001, a média de 28,9 anos, o que 


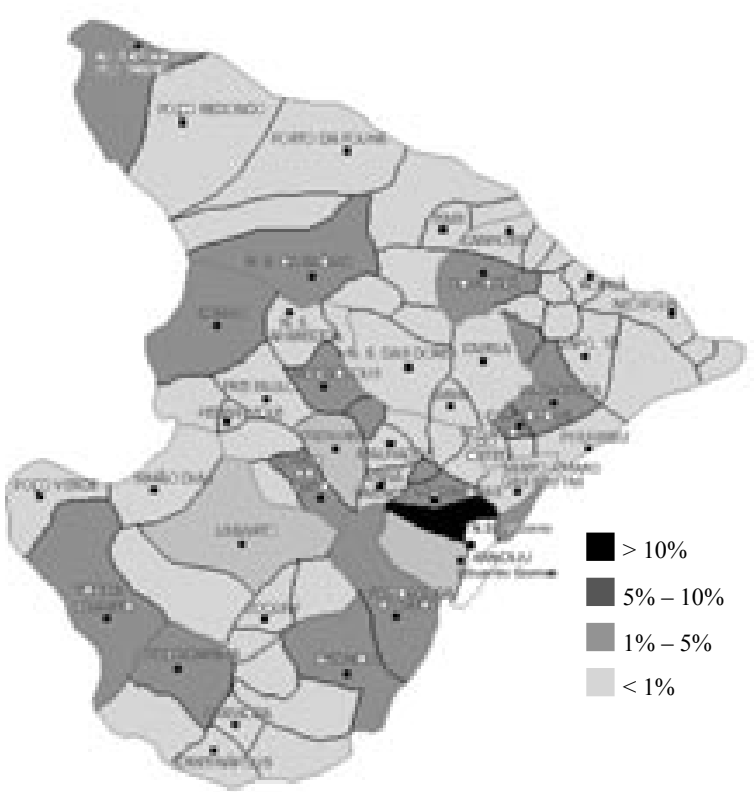

Figura 1 - Cidades de origem dos TCE.
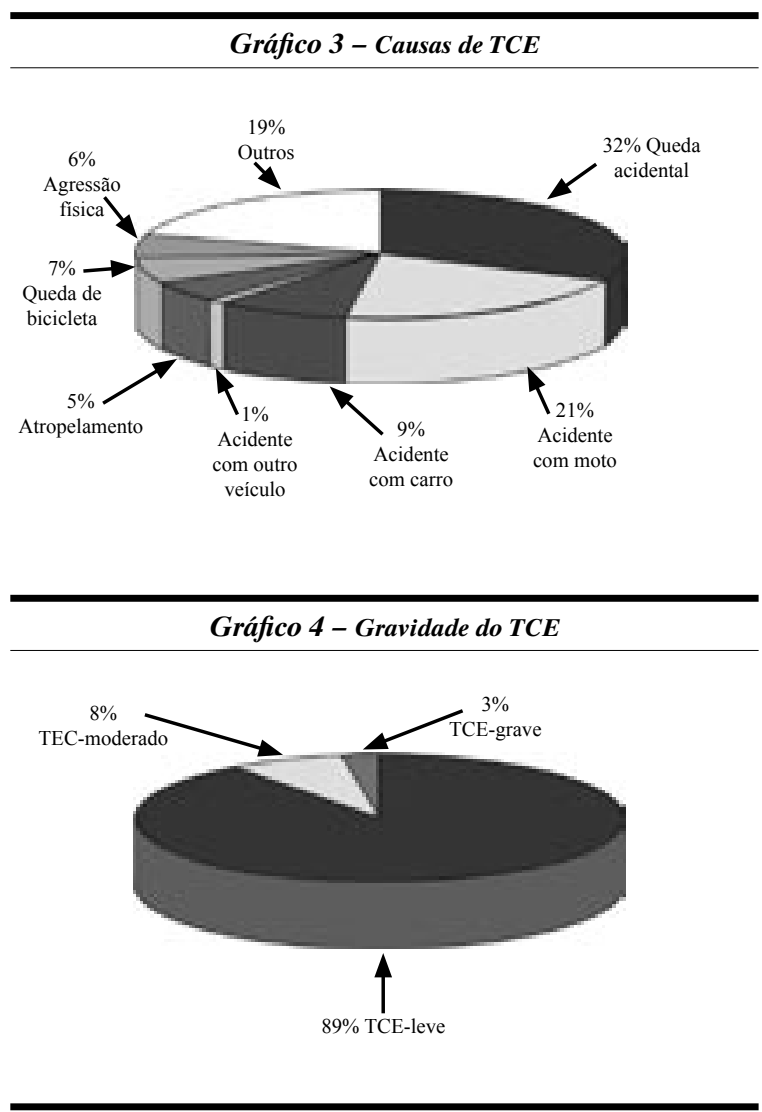

Gráfico 5 - Exame neurológico

Tabela 1

Cidades de origem e número de casos.

\begin{tabular}{lclr}
\hline Município & N & Município & N \\
\hline Nossa Senhora do Socorro & 88 & Tomar do Geru & 4 \\
São Cristóvão & 41 & N. Senhora das Dores & 4 \\
Itabaiana & 36 & Poço Redondo & 4 \\
Lagarto & 32 & Pedrinhas & 4 \\
Tobias Barreto & 18 & Moita Bonita & 4 \\
Laranjeiras & 17 & Gararu & 4 \\
Itaporanga d'Ajuda & 16 & Muribeca & 4 \\
Estância & 16 & Própria & 4 \\
Carira & 12 & Pirambu & 4 \\
Ribeirópolis & 10 & São Domingos & 4 \\
Japaratuba & 9 & Maruim & 4 \\
Umbaúba & 8 & Porto da Folha & 4 \\
Salgado & 8 & Graccho Cardoso & 3 \\
Capela & 8 & Boquim & 3 \\
Riachão do Dantas & 7 & Santa Rosa de Lima & 2 \\
Carmópolis & 7 & Pacatuba & 2 \\
Itabaianinha & 7 & Arauá & 2 \\
Monte Alegre & 7 & Telha & 2 \\
Nossa Senhora da Glória & 7 & Siriri & 2 \\
Aquidabã & 6 & Indiaroba & 2 \\
Barra dos Coqueiros & 6 & Neópolis & 2 \\
Macambira & 6 & Frei Paulo & 2 \\
Campo do Brito & 6 & Rosário do Catete & 2 \\
Canidé do São Francisco & 6 & Areia Branca & 2 \\
Poço Verde & 5 & Sto. Amaro das Brotas & 2 \\
Simão Dias & 5 & TOTAL & $\mathbf{4 7 0}$ \\
\hline & & &
\end{tabular}
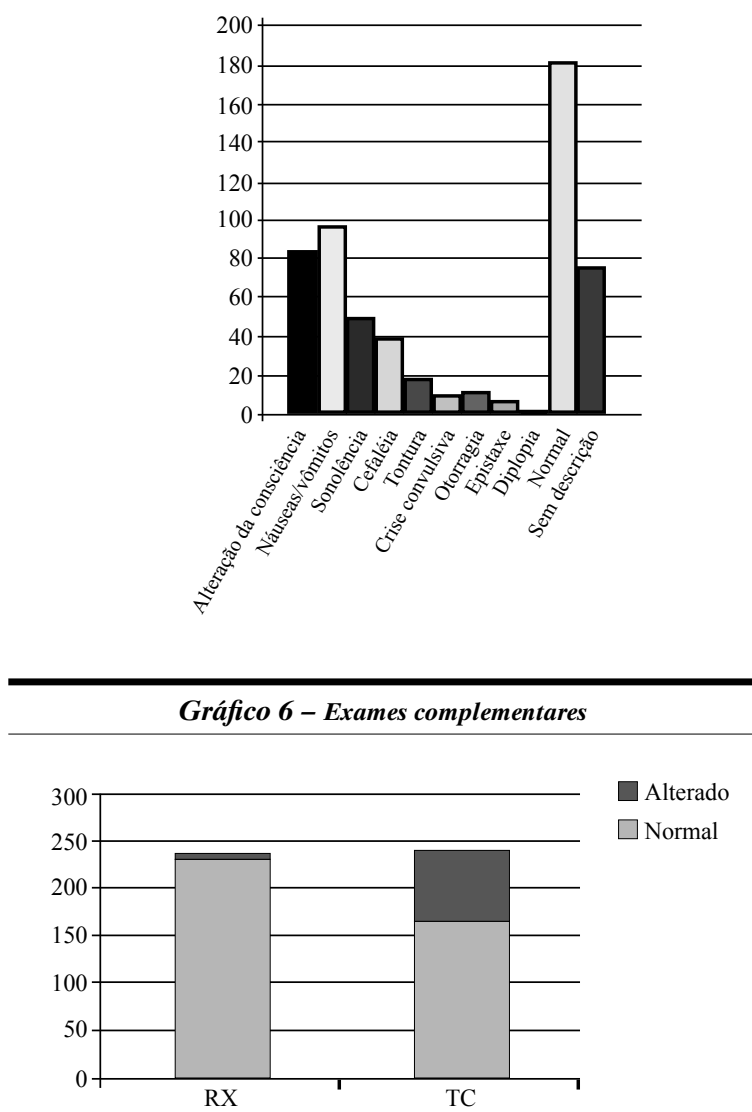
é próximo à faixa etária encontrada em nosso trabalho ${ }^{30}$. Andersson e col. ${ }^{1}$ obtiveram, em seu estudo com 753 pacientes, a média de idade de 27 anos e mediana de 17,5. Observamos também pequeno nivelamento da curva relativa à idade após os 60 anos, que geralmente está relacionado à queda acidental e é compatível com as estatísticas da literatura médica ${ }^{17}$.

O sexo masculino foi o mais acometido, 344 casos $(73 \%)$ e 126 casos (27\%) do sexo feminino (Gráfico 2), dados estes semelhantes aos obtidos na rede Sarah de Hospitais ${ }^{30}$. Anderesson e col. ${ }^{1}$ obtiveram em seu estudo $59 \%$ de pacientes homens e $41 \%$ mulheres, a incidência de 1,46:1. Alguns autores ${ }^{15,17}$ apresentaram a incidência do sexo masculino de 2:1 feminino. Zhao e col. ${ }^{39}$ obtiveram a incidência de 2,5:1. Em nossa casuística, houve a relação de 2,7:1.

Jagger e col. ${ }^{17}$ relataram os três mecanismos mais comuns de TCE na zona rural: acidentes com veículos motorizados, quedas acidentais e violência, fatos estes que foram coincidentes com os nossos achados. Andersson e col. ${ }^{1}$ relataram como causa de TCE em seu estudo, principalmente, quedas acidentais, 58\% dos casos, acidentes com veículos, $16 \%$, e pessoas atingidas por objetos, $15 \%$. O suicídio, apesar de relatado em alguns trabalhos como importante causa de morte por $\mathrm{TCE}^{10}$, não foi relacionado como relevante em nosso trabalho. Em relação ao trauma cerebral decorrente de arma de fogo, sabe-se que $90 \%$ das vítimas morrem no local, e $45 \%$ de todos os acidentes com arma de fogo relacionados à morte foram causadas por $\mathrm{TCE}^{28}$. Geralmente, acidentes com arma de fogo na zona rural estão relacionados à caça ${ }^{28}$.

O TCE pode ser classificado em três categorias segundo sua gravidade: leve, moderado e grave. No grau leve, o paciente pode apresentar-se consciente, sem quaisquer sinais ou sintomas, ou apresentar-se confuso e levemente sonolento, porém, despertando após estímulos apropriados. Nos casos graves, a vítima do traumatismo apresenta-se em coma, sem abertura ocular, sem resposta verbal e motora adequada. Os casos intermediários são classificados como modera$\operatorname{dos}^{38}$. Quanto à gravidade do TCE tivemos 422 (89\%) leve, 36 (8\%) moderado e 12 (3\%) grave (Gráfico 4). A literatura médica relata a incidência de $50 \%$ a $75 \%$ dos casos de TCE-leve ${ }^{38}$.

Com relação ao tratamento e evolução do trauma, 368 pacientes foram liberados para o lar após um determinado período de tempo em observação, 99 foram internados e três evoluíram para óbito (Gráfico 7). Anderesson e col $^{1}$. obtiveram em seu estudo a taxa de mortalidade intra-hospitalar de $0,71 \%$, próximo ao $1 \%$ observado em nossa casuística.

Apesar dessa pesquisa demonstrar que $89 \%$ dos pacientes apresentam TCE-leve e que $78 \%$ dos pacientes

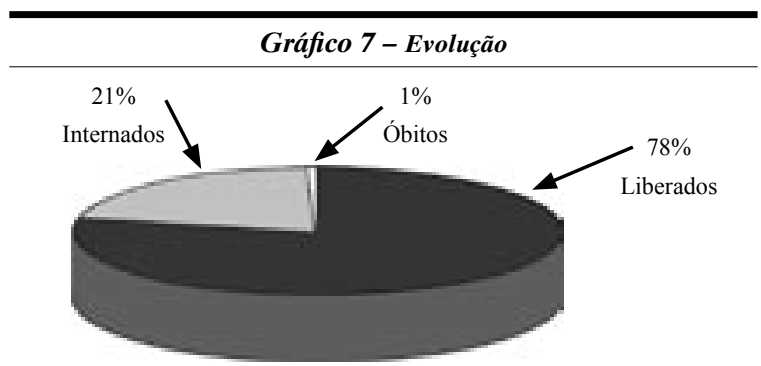

são liberados após atendimento inicial, o TCE na zona rural não deve ser negligenciado, dado a esse trauma representar um grande problema de saúde pública, devido aos altos custos hospitalares com atendimentos, exames complementares, tratamento e reabilitação dos pacientes seqüelados. Nossos resultados fornecem um ponto de partida para a análise das causas e prevenção do TCE na zona rural. Isso apresenta grande potencial na redução tanto das taxas de TCE, quanto na gravidade do trauma ao grupo de risco.

\section{Conclusão}

Do presente trabalho concluímos que:

As principais causas de TCE em residentes da zona rural foram as quedas, seguidas de acidentes com veículos de duas rodas (motos e bicicletas) e agressões físicas.

As faixas etárias mais acometidas foram a segunda e terceira décadas de vida, com uma média de 22 anos e moda de 18 , com variação de idade entre 2 meses e 82 anos.

Houve predomínio do sexo masculino, com uma incidência 2,7:1 do sexo feminino.

Também evidenciamos que os traumas acontecem em sua maioria nas regiões circunvizinhas à capital do Estado, dada a maior densidade populacional destas áreas, principalmente Nossa Senhora do Socorro.

Alterações do nível de consciência e náuseas e/ou vômitos foram os principais achados clínicos.

A TC de crânio apresentou lesão em 75 das 242 solicitadas e a radiografia simples de crânio apresentou alteração em quatro das 239 realizadas.

Receberam alta médica $78 \%$ dos pacientes, $21 \%$ foram internados e $1 \%$ de óbitos.

A observação destes dados sugere que as características dos eventos traumáticos são específicas ao mecanismo de trauma, à faixa etária e ao sexo do paciente, o que reforça a idéia de que traumas são em grande parte previsíveis e não obra do acaso. Estratégias de intervenção devem ser tomadas levando-se em conta essas características. 


\section{Referências}

1. ANDERSSON EH, BJÖRKLUND R, EMANUELSON I, STALHAMMAR D: Epidemiology of traumatic brain injury: a population based study in western Sweden. Acta Neurol Scand 107:256-9, 2003.

2. ANEGERS JF, BRABOW JD, KURLAND LT, LAWS ER: The incidence, causes, and secular trends of head trauma in Olmsted County, Minnesota, 1935-1974. Neurology 30:912-9, 1980.

3. BAETHMANNA, CHAPUIS D, WIRTHA: System analysis of patient management during the pre- and early clinical phase in severe head injury. Acta Neurochir (Wien) 73:93-7, 1999.

4. BROOKS N: Psychological sequelae of head injury. Scott Med J 23:104, 1978.

5. CATLIDGE E: Post concussional syndrome. Scott Med J 23:104, 1978

6. CAVENESS W: Epilepsy, a product of trauma in our time. Epilepsia 17:207-15, 1976

7. COOPER KD, TABADDOR K, HAUSER WA, SHULMAN K, FEINER C, FACTOR PR: The epidemiology of head injury in the Bronx. Neuroepidemiology 2:70-88, 1983.

8. DODGE GG, COGBILL TH, MILLER GJ, LANDERCASPER J, STRUTT PJ: Gunshot wounds: 10-year experience of a rural, referral trauma center. Am Surg 60:401-4, 1994.

9. EVANS CD: Aspects of recovery from physical disability after head injuries. Scott Med J 23:105, 1978.

10. FAHY TJ, IRVING MH, MILLAC P: Severe head injuries - a six year follow-up. Lancet 2:475-9, 1967.

11. FIELD JH: Epidemiology of head injuries in England and Wales. London: Her Majesty's Stationary Office. 1976.

12. FIFE D, FAICH G, HOLLINSHEAD W, BOYNTON W: Incidence and outcome of hospital-treated head injury in Rhode Island. Am J Public Health 76:773-8, 1986.

13. FIFE D: Head injury with and without hospital admission: comparisons of incidence and short-term disability. Am J Public Health 77:810-2, 1987.

14. FRIEDMAN AH: Traumatismo Cranioencefálico. In: Sabiston D.C. Tratado de Cirurgia. Rio de Janeiro: Guanabara Koogan 1999, vol. 2, 1261-6.

15. GABELLA B, HOFFMAN RE, MARINE WW, STALLONES L: Urban and rural traumatic brain injuries in Colorado. Ann Epidemiol 7:207-12, 1997.

16. GENNARIELLI TA, CHAMPION HR, COPES WS, SACCO WJ: Comparison of mortality, morbidity, and severity of 59,713 head-injured patients with 114,447 patients with extracranial injuries. J Trauma 37:962-8, 1994.

17. JAGGER J, LEVINE JL, JANE JA, RIMEL RW: Epidemiologic features of head injury in a predominantly rural population. J Trauma 24:40-4, 1984.

18. JENNETT B, MACMILLAN R: Epidemiology of head injury. Br Med J 282:101-4, 1981.

19. JOHNSTONE B, NOSSAMAN LD, SCHOPP LH, HOLMQUIST L, RUPRIGHT SJ: Distribution of services and supports for people with traumatic brain injury in rural and urban Missouri. J Rural Health. 18:109-17, 2002.

20. KALSBEEK WD, MCLAURIN RL, HARRIS BS, MILLER JD: The national head and spinal cord survey. Major findings. J Neurosurg 53:S19-S31,1980.

21. KESSELA, NICHOLSONA, GRAVES G, KRUPINSKI J: Suicidal attempts in an outer region of metropolitan Melbourne and in a provincial region of Victoria. Aust NZ J Psychiatr 9:255-61, 1975.
22. KLAUBER MR, BARRETT-CONNOR E, MARSHALL LF, BOWERS SA: The epidemiology of head injury: A prospective study of an entire community - San Diego County, California, 1978. Am J Epidemiol 113:500-9, 1981.

23. KRAUS JF, BLACK MA, HESSOL N, et al.: The incidence of acute brain injury and serious impairment in a defined population. Am J Epidemiol 119:186-201, 1984.

24. KUMARASWAMY N, NAZIAH A, ABDULLAH J, ARIFF MMED AR, ABDULLAH MR, GHAZAIME G: Outcome of children with traumatic brain injury in rural Malaysia. J Clin Neurosci 9:251-5, 2002.

25. MACKENZIE EJ, EDELSTEIN SL, FLYNN JP: Trends in hospitalized discharge rates for head injury in Maryland, 1979-86. Am J Public Health 80:217-9, 1990.

26. MUELLEMAN RL, WALKER RA, EDNEY JA: Motor vehicle deaths: a rural epidemic. J Trauma 35:717-9, 1993.

27. NEUROLOGY CHANNEL: [on line] <http://www.neurologychannel.com/tbi/>.

28. PIKUS HJ, BALL PA: Characteristics of cerebral gunshot injuries in the rural setting. Neurosurg Clinics North Am 6: 611-20, 1995

29. REID SR, ROESTER JS, GAICHAS AM, TSAI AK: The epidemiology of pediatric traumatic brain injury in Minnesota. Arch Pediatr Adolesc Med 155:784-9, 2001.

30. SARAH HOSPITAIS: [on line] <http://www.sarah.br>.

31. SCHOOTMAN M, FUORTES L: Functional status following traumatic brain injuries: population-based rural-urban differences. Brain Inj 13:995-1004, 1999.

32. SIMPSON D, NORTH JB, GILLIGAN F: Neurological injuries in South Australia: the influence of distance on management and outcome. Aust NZ J Surg. 1984.

33. SIMPSON DA, WORTH RJ: Neurotrauma in country hospitals: the role of computerized tomography scanning. Aust NZ J Surg 59:1-3, 1989.

34. STEADMAN JH, GHAHAM JG: Rehabilitation of the brain injured. Proc Roy Soc Med 63:23-8, 1970.

35. TAYLOR R, HERRMAN H, PRESTON G: Occupation and mortality in Australia: working age males, 1975-77. Health Commission of Victoria and Department of Social and Preventive Medicine. Monash University. 1983.

36. TIRET L, HAUSHERR E, THICOIPE M, et al.: The epidemiology of head trauma in Aquitaine (France), 1986: A community-based study of hospital admissions and deaths. Int J Epidemiol 19:133-40, 1990.

37. WOODWARD A, DORSCH MM, SIMPSON D. Head injuries in country and city: A study of hospital separations in South Australia. Med J Australia 141:13-7, 1984.

38. ZERATI E: [on line] <http://www.neurologiaonline.com. $\mathrm{br} /$ zerati/neuro/trauma.htm>.

39. ZHAO YD, WANG W. Neurosurgical trauma in people's Republic of China. World J Surg 25:1202-4, 2001.

Original recebido em outubro de 2005

Aceito para publicação em janeiro de 2006

\section{Endereço para correspondência:}

Carlos Umberto Pereira

Av. Augusto Maynard, 245/404

49015-380 - Aracaju, SE

email : umberto@infonet.com.br 